Research Article

\title{
In Silico Analysis of the Gene Expression Patterns between Aldosterone-Producing Adenoma and Nonfunctional Adrenocortical Adenoma
}

\author{
Yongfa Dai $\mathbb{D}^{1},{ }^{1}$ Jing Li $\left(\mathbb{D},{ }^{2}\right.$ Hong Wen, ${ }^{3}$ Jie Liu $\left(\mathbb{D},{ }^{1}\right.$ and Jianling Li $\mathbb{D}^{3,4}$ \\ ${ }^{1}$ Department of Cardiovascular Medicine, The First Nanning People's Hospital, Nanning, Guangxi 530016, China \\ ${ }^{2}$ Department of Nuclear Medicine, The First Affiliated Hospital of Guangxi Medical University, Nanning, Guangxi 530031, China \\ ${ }^{3}$ Department of Cardiovascular Medicine, The First Affiliated Hospital of Guangxi Medical University, Nanning, \\ Guangxi 530021, China \\ ${ }^{4}$ Postdoctoral Mobile Station of Guangxi Medical University, Nanning, Guangxi 530021, China
}

Correspondence should be addressed to Jie Liu; gxnnliujie@163.com and Jianling Li; at5056@163.com

Received 8 April 2021; Accepted 15 September 2021; Published 4 October 2021

Academic Editor: Hafiz Ishfaq Ahmad

Copyright (C) 2021 Yongfa Dai et al. This is an open access article distributed under the Creative Commons Attribution License, which permits unrestricted use, distribution, and reproduction in any medium, provided the original work is properly cited.

\begin{abstract}
Primary aldosteronism is the most common form of secondary hypertension, and aldosteronoma makes up a significant proportion of primary aldosteronism cases. Aldosteronoma is also called aldosterone-producing adenoma (APA). Although there have been many studies about APA, the pathogenesis of this disease is not yet fully understood. In this study, we aimed to find out the difference of gene expression patterns between APA and nonfunctional adrenocortical adenoma (NFAA) using a weighted gene coexpression network (WGCNA) and differentially expressed gene (DEG) analysis; only the genes that meet the corresponding standards of both methods were defined as real hub genes and then used for further analysis. Twenty-nine real hub genes were found out, most of which were enriched in the phospholipid metabolic process. WISP2, S100A10, SSTR5-AS1, SLC29A1, $A P O C 1$, and SLITRK4 are six real hub genes with the same gene expression pattern between the combined and validation datasets, three of which indirectly or directly participate in lipid metabolism including WISP2, S100A10, and APOC1. According to the gene expression pattern of DEGs, we speculated five candidate drugs with potential therapeutic value for APA, one of which is cycloheximide, an inhibitor for phospholipid biosynthesis. All the evidence suggests that phospholipid metabolism may be an important pathophysiological mechanism for APA. Our study provides a new perspective regarding the pathophysiological mechanism of APA and offers some small molecules that may possibly be effective drugs against APA.
\end{abstract}

\section{Introduction}

Primary aldosteronism (PA) is now widely recognized as the common causative agent of hypertension cases, especially for treatment-resistant hypertension [1]. Compared to essential hypertension, PA increases the risk of cardiovascular diseases such as stroke, coronary artery disease, atrial fibrillation, and heart failure [2]. The currently accepted prevalence rate of PA is $5-10 \%$ among hypertension patients, but the actual prevalence rate might be 3-5 times higher than the currently supposed rate [3]. PA is mainly caused by aldosterone-producing adenoma (APA) [4], an adrenal tumor that excessively secretes aldosterone, which leads to hypertension and hyperkalemia. Certain adrenocortical adenomas, called nonfunctional adrenocortical adenoma (NFAA), do not secrete hormones but simply manifest as a small lump on the upper pole of the kidney. A nationwide multicenter study in Japan revealed that $50.8 \%$ of total adrenal incidentalomas were NFAA, while only $5.1 \%$ were APA [5]. This study aimed to find out the differences of the gene expression pattern between these kinds of states which may let us further understand the pathogenesis of APA. Previous studies have identified somatic mutations in the potassium channel gene KCNJ5 and the calcium channel gene CACNA1D, which contribute to aldosterone production and cell proliferation in adrenal glomerulosa $[6,7]$. Our 
study mostly focuses on the gene expression level of APA and NFAA. It has been acknowledged that unilateral forms of PA can be treated by adrenalectomy, while bilateral disease only can be treated by medical therapy based on mineralocorticoid receptor antagonists [8]. Since adrenalectomy is not suitable for bilateral APA and mineralocorticoid receptor antagonists are associated with various sexsteroid-related adverse effects, mainly breast tenderness, menstrual abnormalities, decreased libido, and impotence, especially when used at high doses, development of new drugs for the treatment of APA is necessary [9]. According to one of the latest studies, mineralocorticoid receptor antagonists might decrease the rates of positive screening for primary aldosteronism [10]. In this study, we are going to find out some alternative drugs for the treatment of APA according to its gene expression pattern. Hence, investigating differences between the expression patterns of patients with APA and those with NFAA may improve our understanding of the pathogenesis of this disease and provide new clues for the treatment of APA. The workflow of the study is shown in Figure 1.

\section{Materials and Methods}

2.1. Microarray Data Information and Data Preprocessing. Raw microarray data (GSE33371, GSE28476, and GSE10927) of the Affymetrix platform (GPL570) were downloaded from the GEO database [11-13]. Twentyeight adrenocortical adenoma samples from GSE33371 and GSE28476 were integrated as a combined dataset for further analysis, including 10 samples of APA, 12 samples of cortisol-producing adrenocortical adenoma (CPA), and 6 samples of NFAA. Thirteen adrenocortical adenoma samples from GSE10927 were used as a validation dataset. All analyses were performed on R software (version 4.0.0). Probe sets were annotated using the getGEO function of the GEOquery package. Prior to differential gene expression analysis and weighted gene coexpression network analysis (WGCNA), probes matching more than one gene were removed, and multiple probes matching one gene were then used for calculating the average expression value.

2.2. Identification of DEGs. Both the combined and validation datasets underwent differential expression gene (DEG) analysis. The Robust Multiarray Average algorithm was used to normalize the raw data [14]. The ComBat function of the sva package was applied to eliminate batch effects between GSE33371 and GSE28476 [15]. Principal component analysis (PCA) was used to display the gene expression pattern among CPA, APA, and NFAA of the combined dataset. DEGs analysis were performed on APA vs. NFAA using the limma package with the empirical Bayes method, and those genes with adjusted $P$ value $<0.05$ and | $\log 2$ (fold change) $\mid \geq 1$ were defined as statistically significant DEGs. $P$ values were adjusted using the Benjamini-Hochberg false discovery rate (FDR).
2.3. WGCNA Network Construction. The combined dataset was used for construction of a WGCNA network. The coexpression network was constructed using "WGCNA," an $\mathrm{R}$ package for weighted correlation network analysis [16]. The soft thresholding power was calculated using the pickSoftThreshold function of the WGCNA package. To produce a scale-free network, we used a $\beta$ value $=6$. A onestep network construction method was used to identify coexpression modules using the blockwiseModules function. The plotDendroAndColors function of the WGCNA package was used to visualize the coexpression modules.

\subsection{Construction of Module-Clinical Trait Relationships.} After the coexpression modules were identified, the module eigengene (ME) was calculated using the first principal component of gene expression level in the corresponding module. Module-clinical trait relationships were assessed using the correlations between MEs and interesting clinical traits including APA, CPA, NFAA, adrenocortical adenoma with $\beta$ catenin mutation, and wild-type adrenocortical adenoma. Module membership (MM) and gene significance (GS) were the two important indices used for determining whether a gene was crucial in each module. Genes with | $\mathrm{MM} \mid \geq 0.8$ and $|\mathrm{GS}| \geq 0.2$ were defined as hub genes of the corresponding module. The brown module contained hub genes with the highest correlation coefficient with APA, while the tan module contained hub genes with the lowest correlation coefficient with APA (Figure 2(c)).

\subsection{Identification and Functional Enrichment Analysis of Real} $H u b$ Genes. Real hub genes were defined as the common genes among DEGs of the combined dataset, hub genes of the brown module and hub genes of the tan module. Real hub gene integration networks were constructed using Cytoscape (version 3.8.0) [17], based on their interrelationship in the WGCNA network. Functional enrichment analysis on the real hub genes was conducted using the clusterProfiler package and Metascape database [18]. Enrichment items with adjusted $P$ values $<0.05$ were considered statistically significant.

2.6. Gene Set Enrichment Analysis (GSEA). We used GSEA, a knowledge-based approach for interpreting genome-wide expression profiles, to analyze the gene expression pattern of the combined datasets [19]. Two groups were examined: APA and NFAA. Only Gene Ontology (GO) or Kyoto Encyclopedia of Genes and Genomes (KEGG) annotations with a nominal $P<0.05$ were used for further analysis.

2.7. Validation Using Other Datasets. Thirteen adrenocortical adenoma samples from GSE10927 were used as the validation dataset. Of these samples, six exhibited hyperaldosteronism and the remaining seven behaved as adrenal masses, without hyperaldosteronism or Cushing syndrome. The expression patterns of the real hub genes in the validation dataset were also identified using DEG analysis. When the DEGs analysis of the validation was performed, 

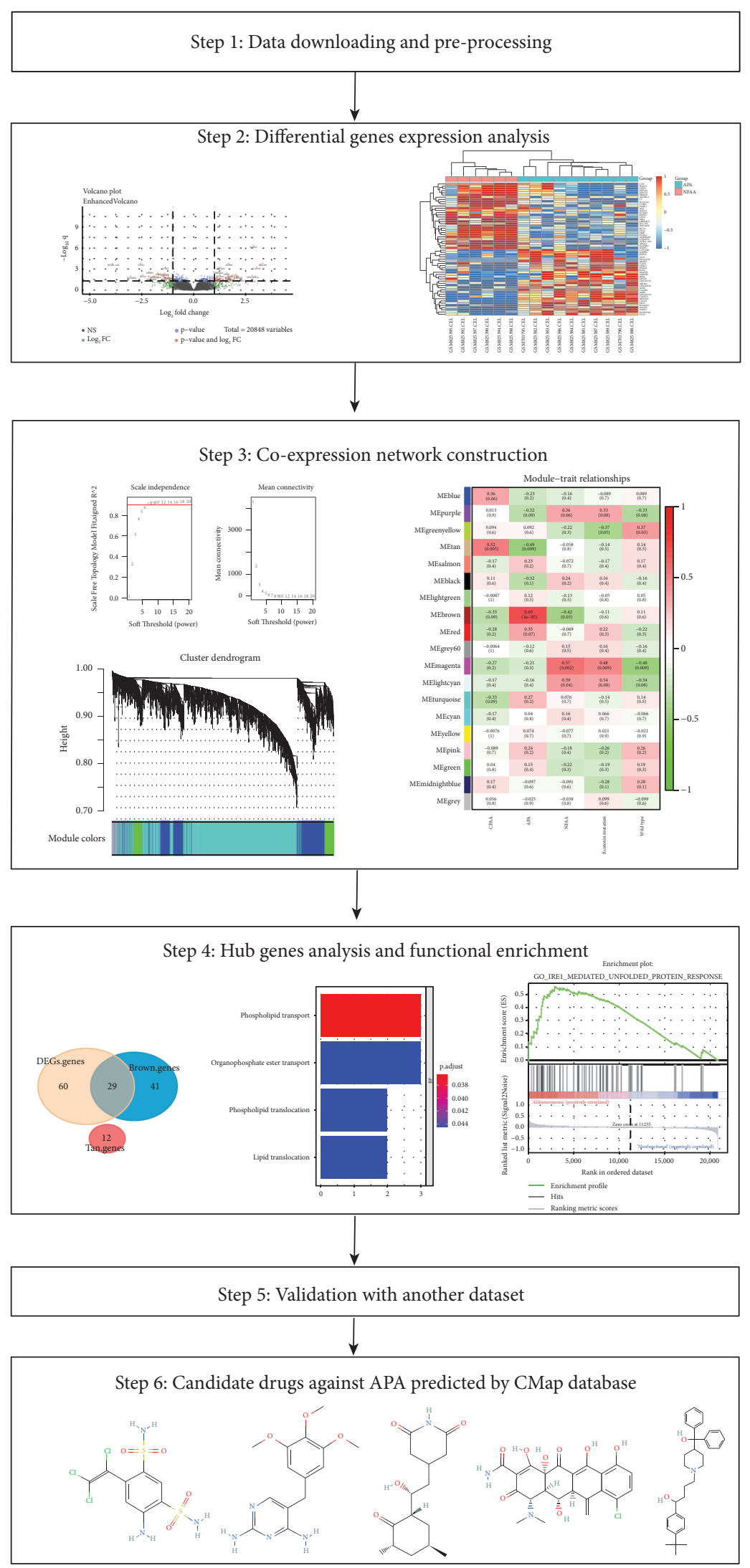

FIgURE 1: Workflow of the study. 

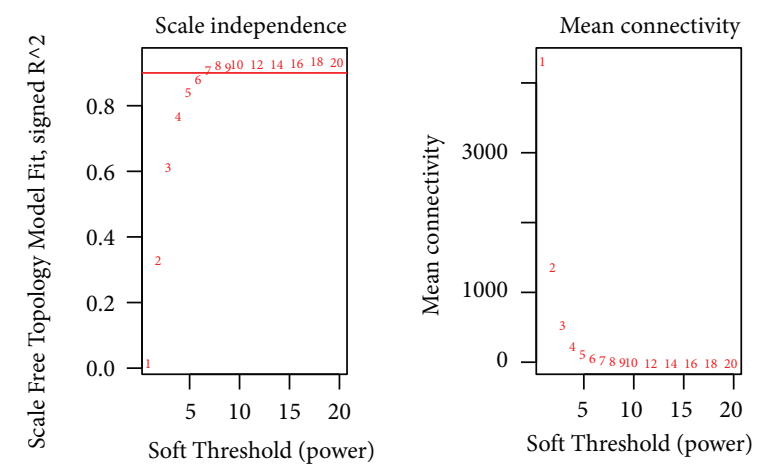

(a)

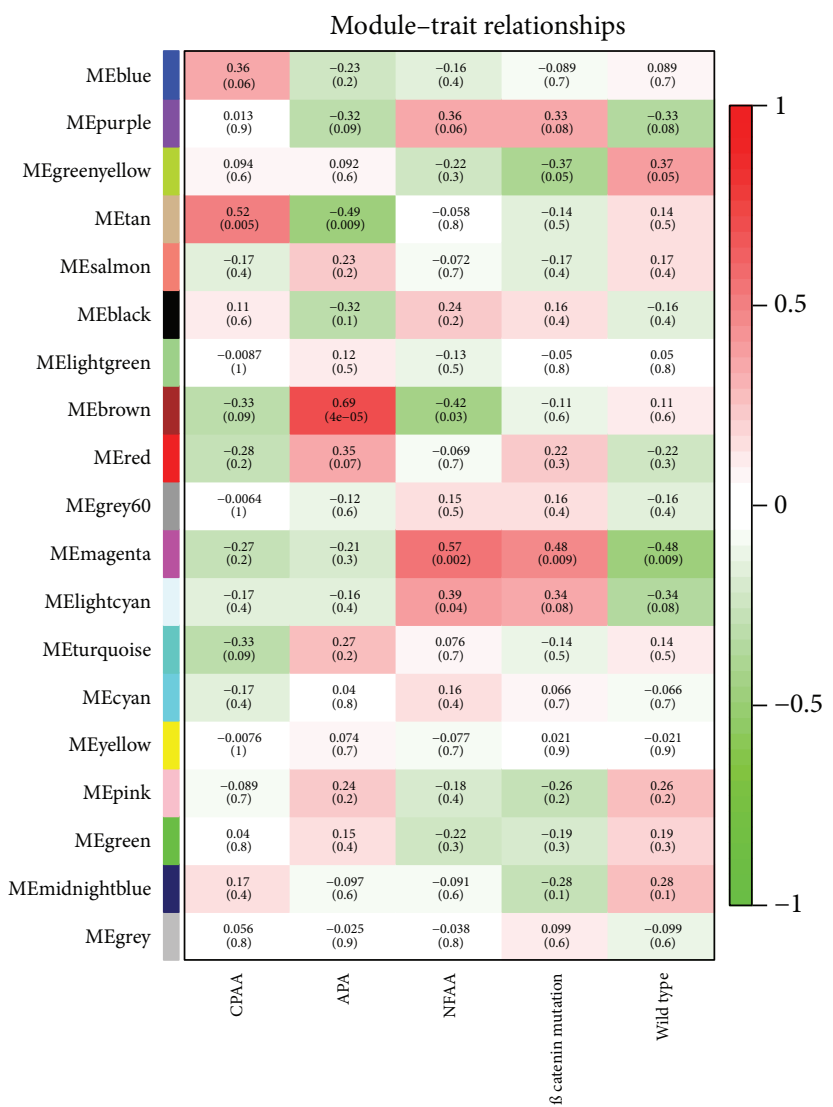

(c)

Module membership vs. gene significance

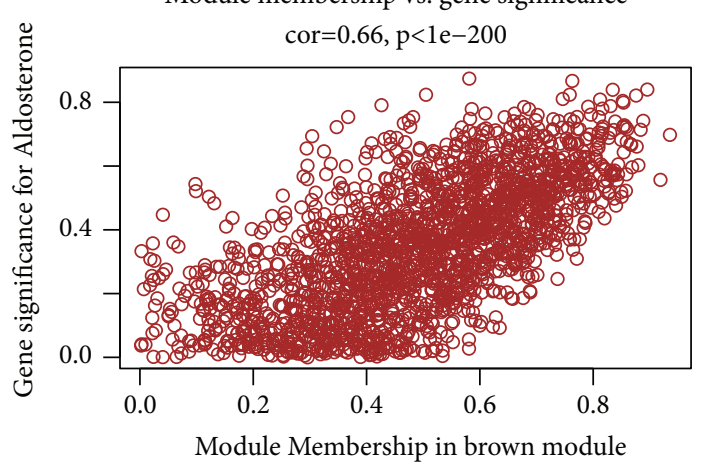

(e)

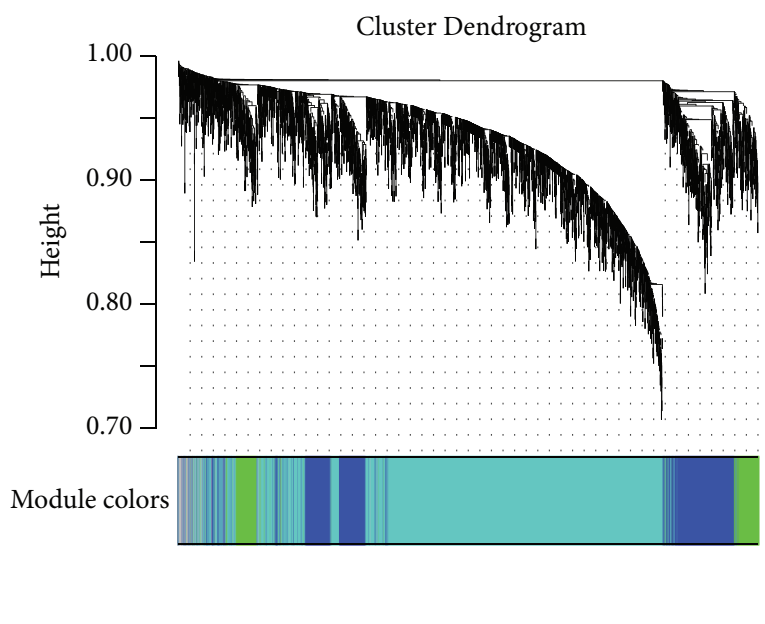

(b)
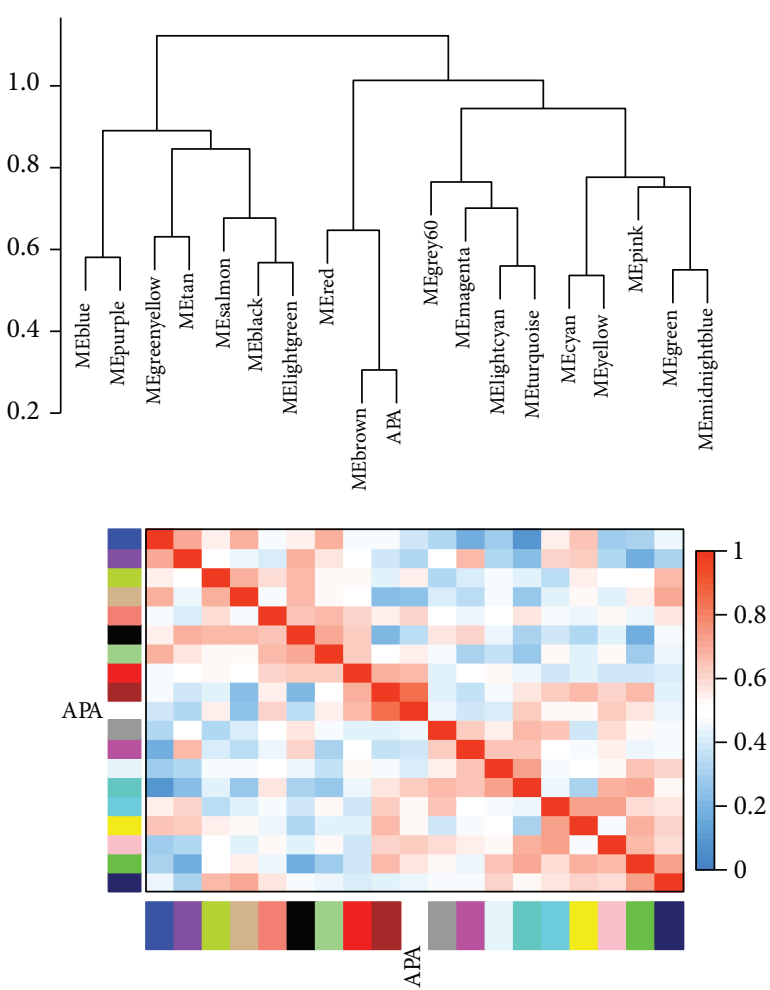

(d)

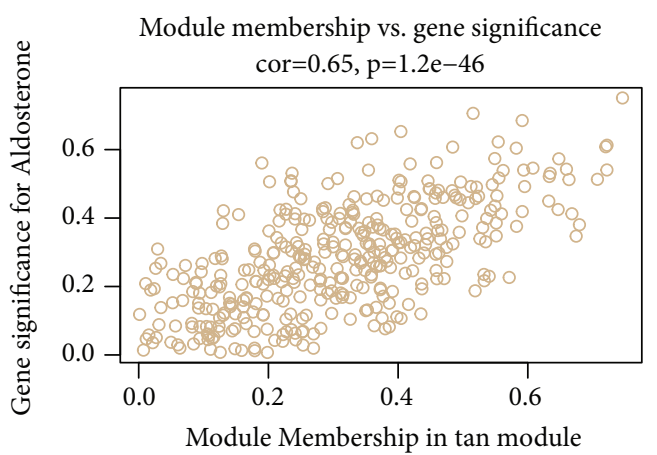

(f)

Figure 2: Continued. 


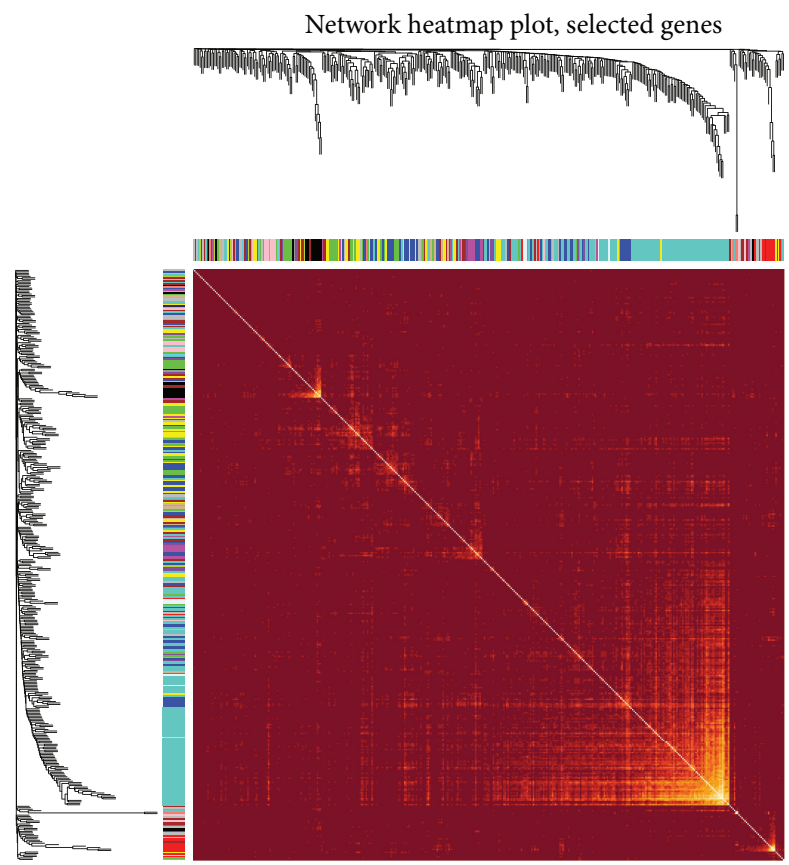

$(\mathrm{g})$

FIGURE 2: WGCNA analysis of combined datasets. (a) Scale independence and mean network connectivity for soft thresholding powers ( $\beta$ ). $\beta=6$ was selected to achieve model fit maximization. (b) In total, 22,848 genes were assigned to 19 modules, including one gray module, with cutoff of powers $=6$. The top image shows a gene dendrogram, and the bottom image shows the gene modules with different colors. (c) Correlation between modules and traits. The upper number in each cell refers to the correlation coefficient of the corresponding module with clinical trait, and the lower number is the corresponding $P$ value. (d) The eigengene dendrogram and heat map. The 19 modules are relatively independent, and the brown module was strongly correlated with APA. (e), (f) Scatter plots of GS versus MM for APA in the brown and tan modules. (g) Topological overlap matrix heatmap of 400 genes selected from a total of 22,848 genes. Light color represents high overlap of the genes, and progressively darker color indicates lower overlap. Blocks of light colors along the diagonal represent the coexpression modules.

the samples exhibiting hyperaldosteronism were defined as the APA group and the samples behaving as adrenal masses were defined as the NFAA group. The expression patterns of the real hub genes in the validation dataset were compared with those of the combined datasets to identify genes with the same expression pattern in both datasets. The results were presented visually using the GraphPad Prism 8 software.

2.8. Identification of Potential Candidate Drugs for the Treatment of APA. Putative small-molecule drugs were predicted using the Connectivity Map (CMap) version build 02 (https://portals.broadinstitute.org/cmap/), a database that documents gene expression profiles from cultured human cells treated with bioactive small molecules, which can be used to find out connections between diseases with a particular gene expression pattern and small-molecule drugs that can counteract the diseases in the gene expression level [20]. The DEGs of the combine datasets were used to query the CMap database. The enrichment scores represent the similarity (ranging from -1 to 1 ) of the gene expression pattern between APA and the cultured human cells treated with the corresponding drugs, a drug inducing similar gene expression pattern with APA will be given a positive score, while a drug producing an opposite pattern will be given a negative score. Hence, a drug with a negative enrichment score was considered to be a putative therapeutic drug for APA.

\section{Results}

3.1. Identification of DEGs in the Combined Datasets. After data preprocessing, PCA was used to analyze the gene expression patterns of the combined datasets. The gene expression pattern of APA was obviously different from that of NFAA, as the spots of APA were spatially far away from NFAA (Figure 3(a)). Eighty-nine DEGs were identified using a cutoff criterion: adjusted $P$ value $<0.05$ and $\mid \log 2$ (fold change) $\mid \geq 1$. These DEGs included 52 genes upregulated and 47 genes downregulated in the APA as compared with NFAA. A volcano plot shows all the differently expressed genes between APA and NFAA (Figure 3(b)). The heat map including the 50 most upregulated and downregulated genes clearly shows the difference between APA and NFAA (Figure 3(c)).

3.2. Coexpression Analysis. After normalization and batch effect elimination, the combined dataset was used for WGCNA. The scale independence and mean network connectivity for soft thresholding powers $(\beta)$ are shown in 

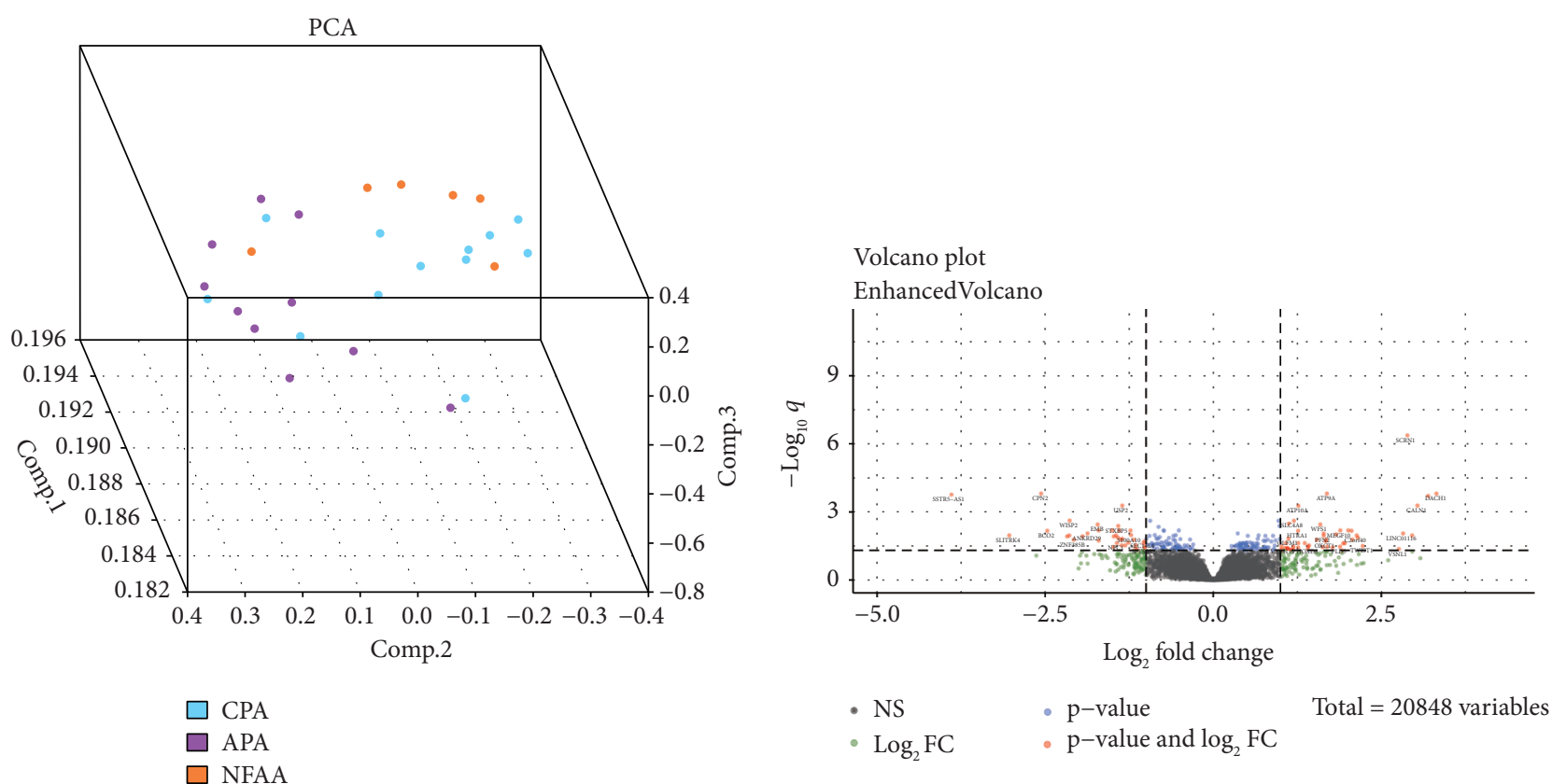

(a)

(b)

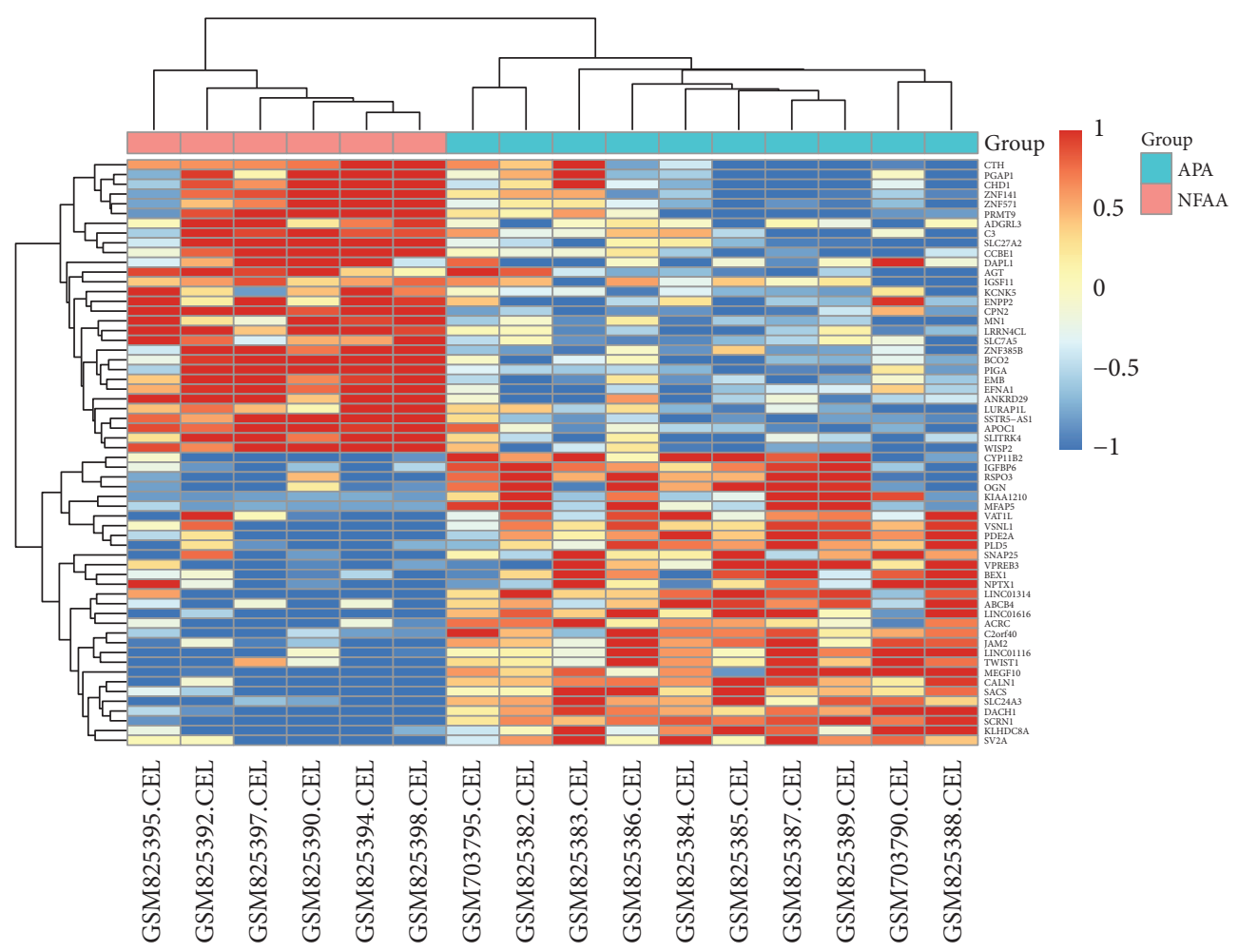

(c)

FIGURE 3: Screening of differentially expressed genes (DEGs) in combined datasets. (a) Spatial graph for PCA of the combined dataset. The light blue spots represent CPA, the purple spots represent APA, and the yellow spots represent NFAA. (b) Volcano map of differently expressed genes between APA tissues and NFAA tissues. (c) Heat map of the top 100 DEGs between the APA and NFAA.

Figure 2(a). $\beta=6$ was selected to maximize the model fit, and 19 modules were identified, including one gray module (Figure 2(b)). Of all those modules, the brown module exhibited the highest positive correlation with APA (module-trait weighted correlation $=0.69)$, whereas the tan module showed the strongest negative correlation with the APA (module-trait weighted correlation $=-0.49$ ). We also observed that the tan module correlated most strongly with $\mathrm{CPA}$, whereas the magenta module correlated most strongly with adrenocortical adenoma with mutations of the 
$\beta$-catenin gene, which occur frequently in adrenocortical adenoma and carcinomas.

An eigengene dendrogram and heat map were used to quantify module similarity and correlation with the clinical trait of APA (Figure 2(d)). The results suggested that there was a high level of independence among the modules. Both the brown and tan modules were selected, and the relationship between GS and MM for APA in both modules is shown on the scatterplot (Figures 2(e) and 2(f)). Genes in both modules highly correlated with APA. For the brown module, $P<1 e-200$ and correlation $=0.66$; for the $\tan$ module, $P<1.2 e-46$ and correlation $=0.65$. Seventy genes from the brown module and 12 genes from the tan module were extracted as hub genes for further analysis, using the criteria $M M \geq 0.8$ and $G S \geq 0.2$. Finally, 400 genes were selected at random from a total of 20,848 genes to construct a network heatmap plot (Figure 2(g)).

\subsection{Identification and Functional Enrichment Analysis of Real} $H u b$ Genes. The real hub genes were an intersection of genes set among DEGs of the combined dataset, hub genes in the brown module and hub genes in the tan module. Twentynine real hub genes were confirmed (Figure 4(a)), including three IncRNA genes (SSTR5-AS1, LINC01314, and LINC01116) and one transcription factor (DACH1). An integration network of the real hub genes was extracted from the coexpression network using Cytoscape software (Figure 4(b)). To evaluate the function of these genes, GO functional enrichment analyses were performed using the clusterProfiler package and the Metascape database (Figures 4(c) and 4(d)). Four biological processes that met the selection criteria (Section 2) were filtered out, GO: 0015914 (phospholipid transport), GO:0015748 (organophosphate ester transport), GO:0045332 (phospholipid translocation), and GO:0034204 (lipid translocation), using the clusterProfiler package; similarly, the enrichment results from the Metascape database were as follows: GO:0015914 (phospholipid transport), GO:0051099 (positive regulation of binding), GO:0016042 (lipid catabolic process), and GO: 0001666 (response to hypoxia). We constructed a GO enrichment network on Cytoscape using the BiNGO plug-in module in which nodes with yellow color were significant enrichment GO items (Figure 5).

3.4. GSEA Results. We performed GSEA for the combined datasets. For GO enrichment analysis, 39 gene sets were enriched in the APA phenotype with $P$ values $<0.01$, whereas 58 gene sets were enriched in the NFAA phenotype with $P$ values $<0.01$. As for KEGG pathway enrichment analysis, four gene sets were enriched in the APA phenotype with $P$ value $<0.05$, whereas seven gene sets were enriched in the NFAA phenotype with $P$ value $<0.05$. Partial results are shown in Figures 6 and 7. GO enrichment analysis revealed that the datasets "negative regulation of lipid localization," "negative regulation of lipid transport," and "plasminogen activation" were enriched in the NFAA phenotype, whereas the datasets "IRE-mediated unfolded protein response," "peptidyl asparagine modification," and "TAU protein kinase activity" were enriched in the APA phenotype. In the KEGG pathway enrichment analysis, the datasets "ABC transporters," "complement and coagulation cascades," and "glycosylphosphatidylinositol GPI anchor biosynthesis" were enriched in the NFAA phenotype, whereas the datasets "glycerophospholipid metabolism," "GnRH signaling pathway," and "linoleic acid metabolism" were enriched in the APA phenotype.

3.5. Validation of Real Hub Genes Using GSE10927. We used another dataset GSE10927 to validate the real hub genes. Seventeen DEGs of the validation dataset that met the setting criteria (Section 2) were filtered out. The gene expression pattern of these DEGs was compared with that of the previously identified 29 real hub genes of the combined dataset. Six genes-WISP2, S100A10, SSTR5-AS1, SLC29A1, $A P O C 1$, and SLITRK4-with the same expression pattern in the two datasets were identified (Figure 8). All these genes showed relatively low expression in APA samples and high expression in NFAA samples, with all $P$ values $<0.001$.

3.6. Screening of Candidate Small-Molecule Drugs for the Treatment of APA. Under the screening conditions of enrichment scores $<-0.75$ and $P$ value $<0.01$, five candidate drugs with potential therapeutic value for APA were filtered out (Table 1), including clorsulon (C8H8Cl3N3O4S2), trimethoprim (C14H18N4O3), cycloheximide (C15H23NO4), meclocycline (C22H21ClN2O8), and terfenadine (C32H41NO2). All of these drugs showed significantly negative correlations with the gene expression pattern in APA, which indicated that these pharmaceuticals may be effective drugs against APA. We also investigated the $2 \mathrm{D}$ structure of these candidate drugs using the Pubchem database (https://pubchem.ncbi.nlm.nih.gov/), as shown in Figure 9.

\section{Discussion}

In this study, we combined adrenocortical adenoma samples from two series of GEO datasets to perform differential gene expression analysis and coexpression network analysis. A cluster of real hub genes was identified including three lncRNAs (SSTR5-AS1, LINC01314, and LINC01116) and one transcriptional regulator $(D A C H 1)$. These genes may play an important role in regulating the expression of other real hub genes. LINC01116 has been implicated in osteosarcoma, gastric cancer, glioma, and head and neck squamous cell carcinoma, for promoting the proliferation, invasion, and migration of tumor cells [21-23]. We observed that LINC01116 was highly expressed in NFAA samples and lowly expressed in APA samples, suggesting that it is a protective factor for APA. DACH1 as a transcription factor is essential for podocyte function [24]. However, the role of $D A C H 1$ in the pathogenesis of APA requires further investigation.

We performed functional enrichment through various methods to further analyze the real hub genes. Using the clusterProfiler package, we identified four highly correlated 


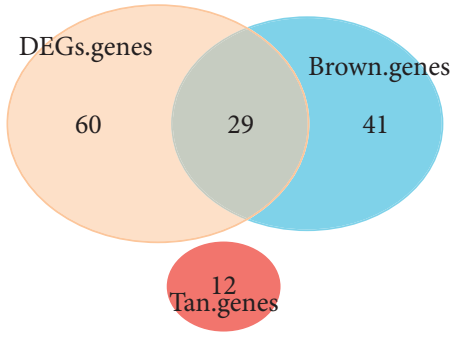

(a)

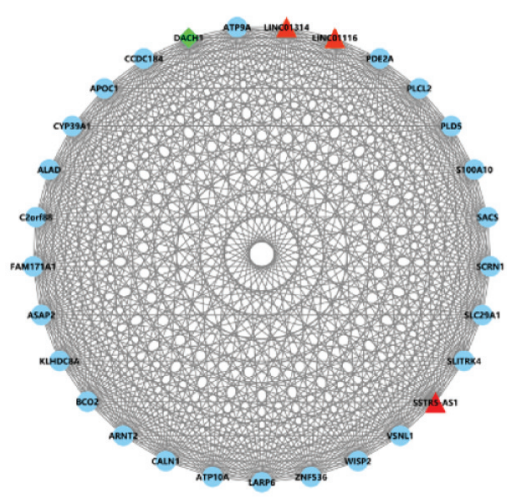

(b)

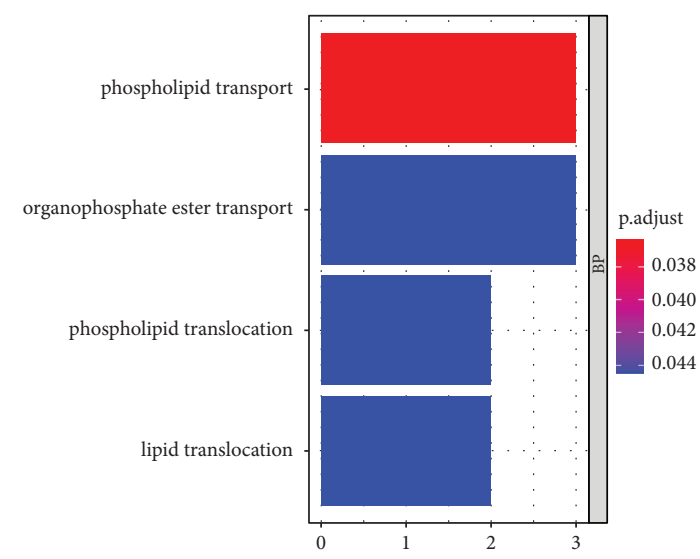

(c)

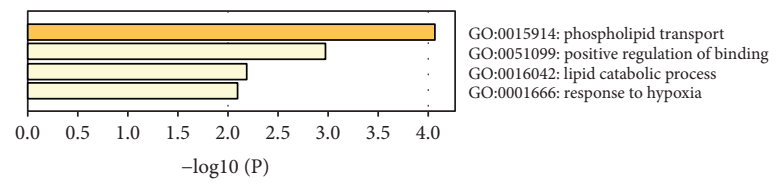

(d)

FIGURE 4: Identification of real hub genes and functional enrichment. (a) The Venn diagram of DEGs of the combined dataset and hub genes from the brown and tan modules. Twenty-nine real hub genes were identified. (b) The interaction network of lncRNA, transcription factor, and coding mRNA for the real hub genes. The red triangles represent lncRNA, the green diamond represents the transcription factor, and the other light blue circles represent the protein coding genes. (c), (d) The results of functional enrichment analysis using the clusterProfiler package and Metascape database. Phospholipid transport was enriched in both methods.

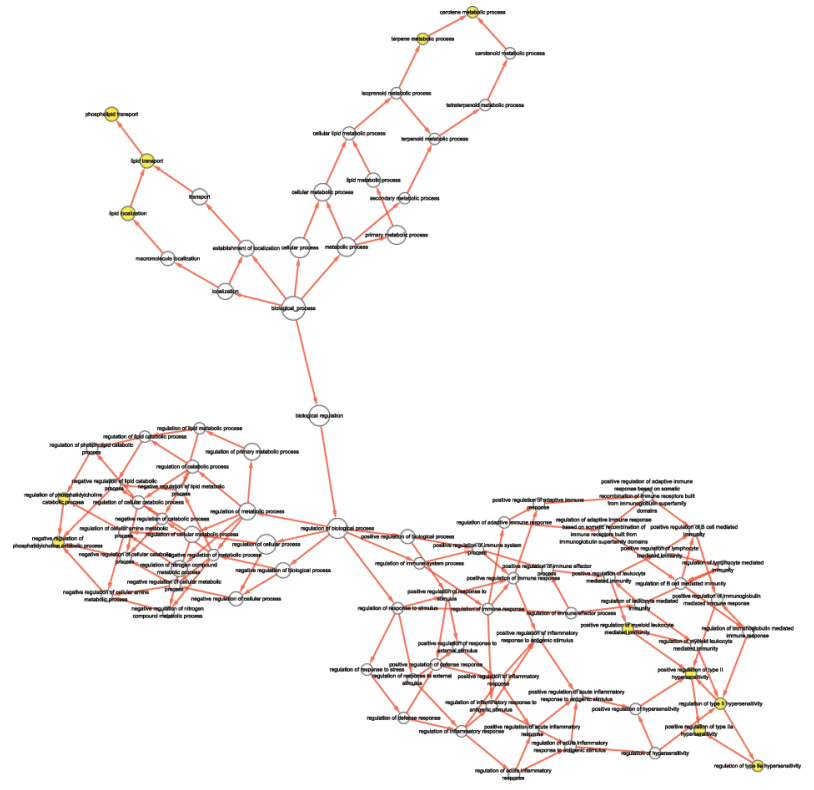

FIGURE 5: Enrichment network of the real hub genes using the BiNGO plug-in module of Cytoscape; nodes with yellow color indicate significant $P$ values for the corresponding GO items; the size of the nodes indicates the number of genes enriched in the corresponding GO items; the bigger the size of the nodes, the more the genes were enriched in the corresponding GO items.

biological processes: phospholipid transport, organophosphate ester transport, phospholipid translocation, and lipid translocation, of which phospholipid transport is the one with the lowest adjusted $P$ value. Phospholipid transport was also found to be a significant enriched item, when using the Metascape database for functional enrichment analysis. After that, GO annotation enrichment was performed on Cytoscape using the BiNGO plug-in module and a GO enrichment network was also constructed. Most significant items in the GO enrichment were related to phospholipid transportation and (or) phospholipid metabolic processes. Taken together, these results suggest that phospholipid transportation and metabolism may be the pathogenesis of APA.

Similar conclusions were obtained from the results of GSEA enrichment analysis. The enrichment results of GO annotations for the NFAA phenotype included "negative regulation of lipid localization" and "negative regulation of lipid transport," whereas the enrichment results of the KEGG pathway for the APA phenotype contained glycerophospholipid metabolism, indicating that a high level of phospholipid metabolic activity in APA may differentiate it from NFAA. The underlying molecular mechanisms require further investigation, although the GSEA enrichment results have provided some clues.

Six genes with the same expression pattern in the combined and validation datasets were identified. However, the validation samples had its limitations. Owing to the absence of data about secretory hormones in the validation samples, sample grouping may yield false negative results when dividing the adrenal mass samples to the NFAA group. The hyperaldosteronism samples were certainly APA; however, we cannot ensure that all the adrenal mass samples were NFAA. Although this phenomenon might generate 
Enrichment plot:

GO_NEGATIVE_REGULATION_OF_LIPID_LOCALIZATION

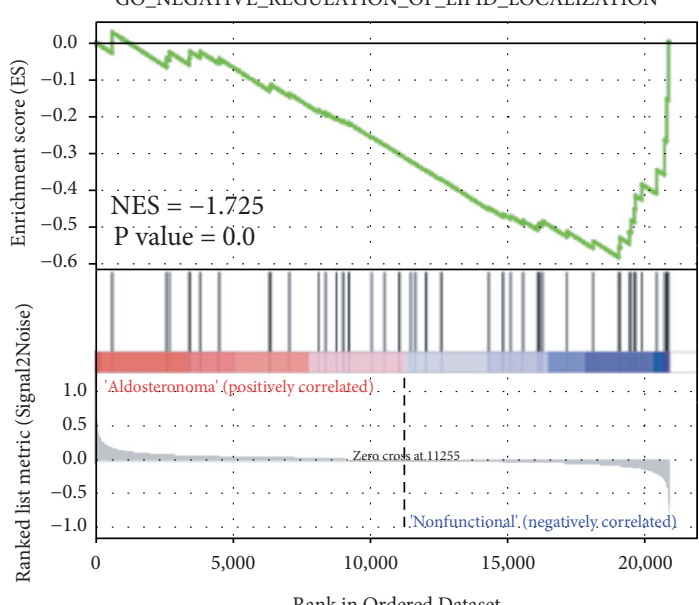

—_ Enrichment profile

_ Hits

Ranking metric scores

(a)

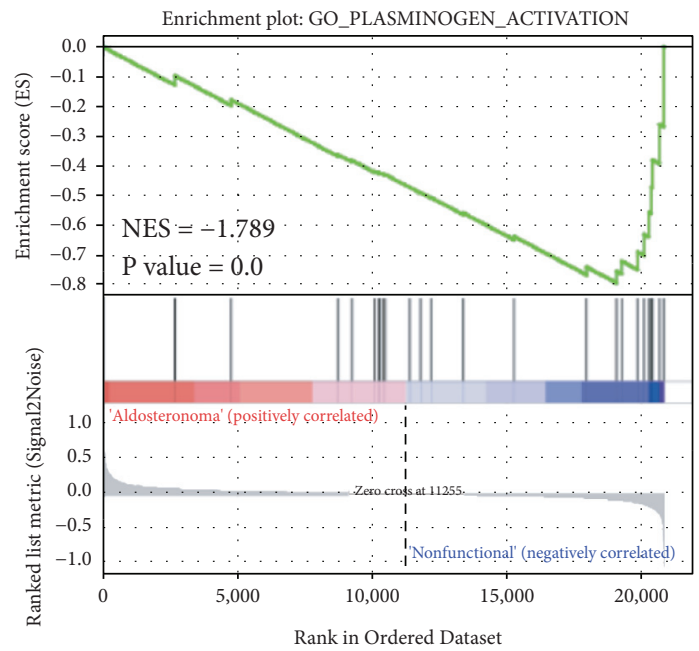

Enrichment profile

- Hits

___ Ranking metric scores

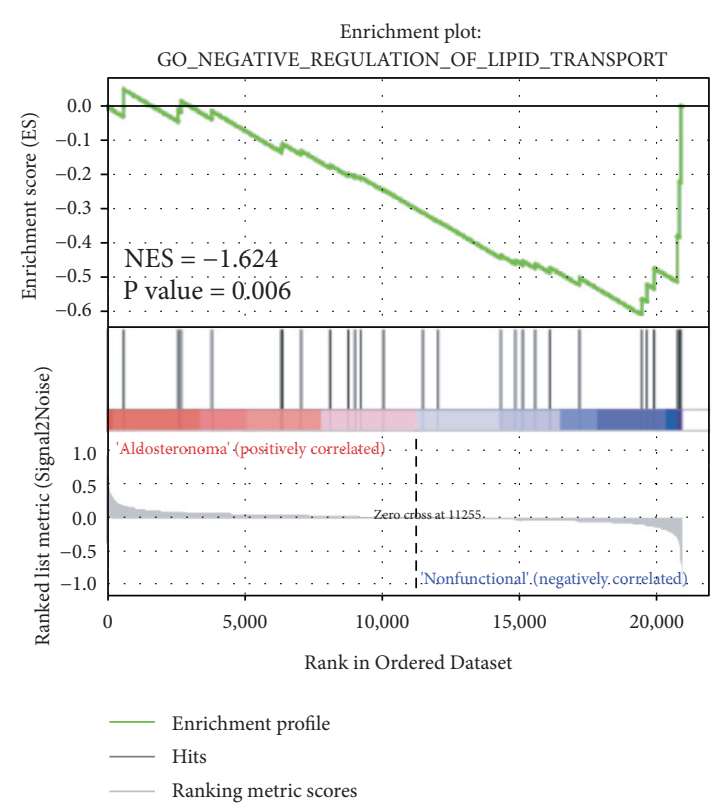

(b)

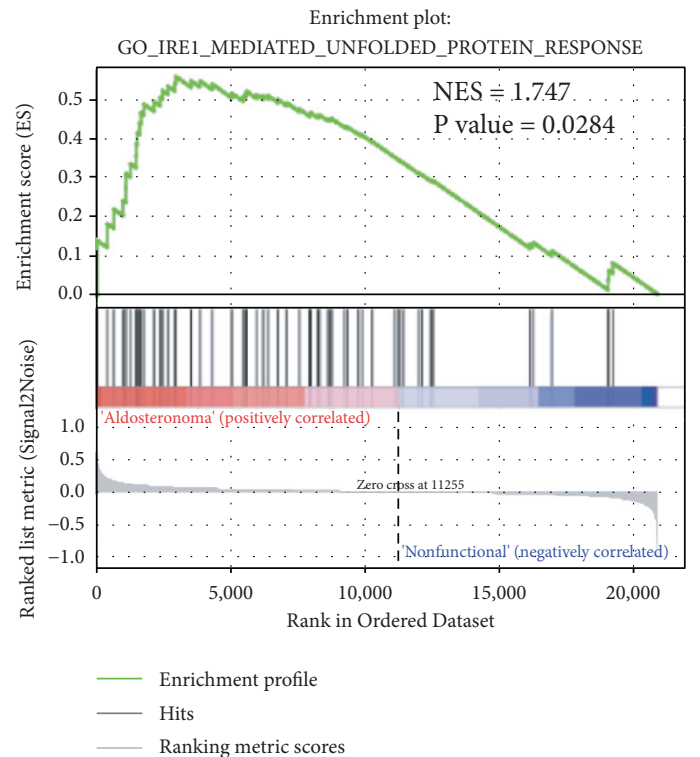

(d)

Figure 6: Continued. 


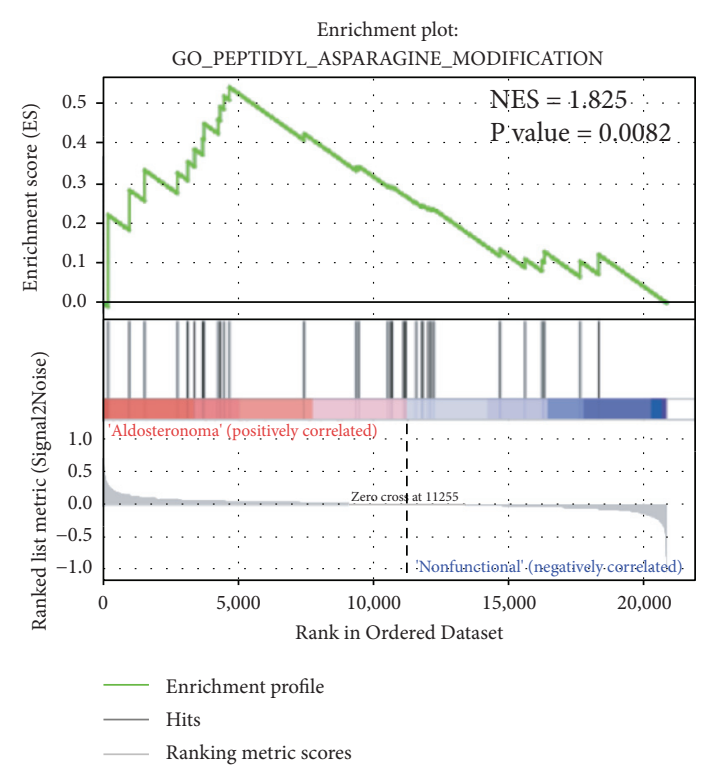

(e)

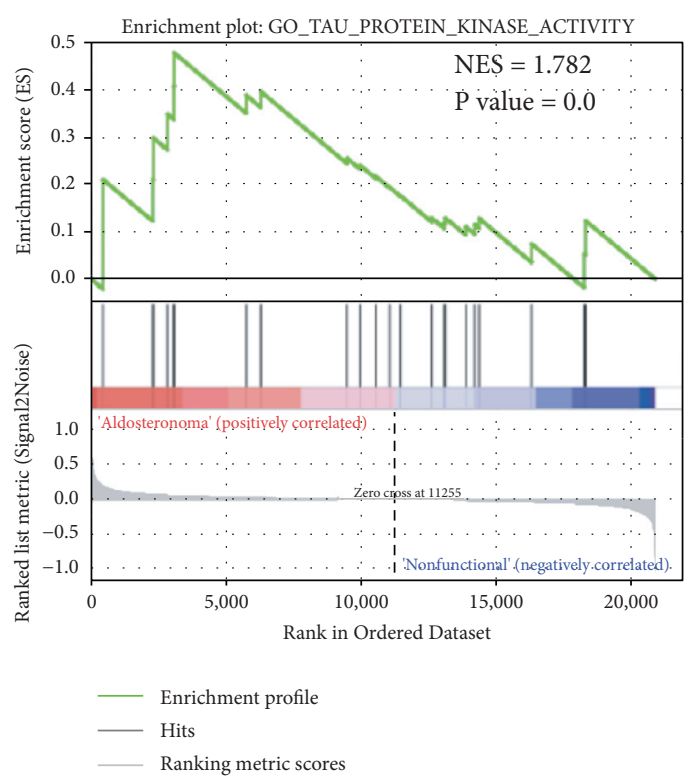

(f)

FIGURE 6: GSEA analysis for GO enrichment using combined datasets. IRE1, an endoplasmic reticulum stress sensor.

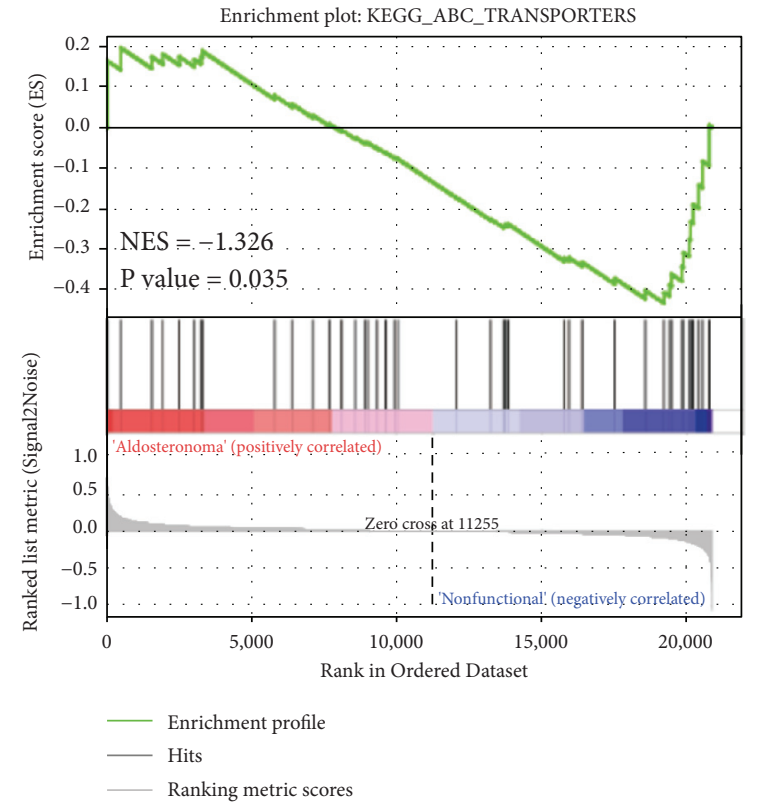

(a)

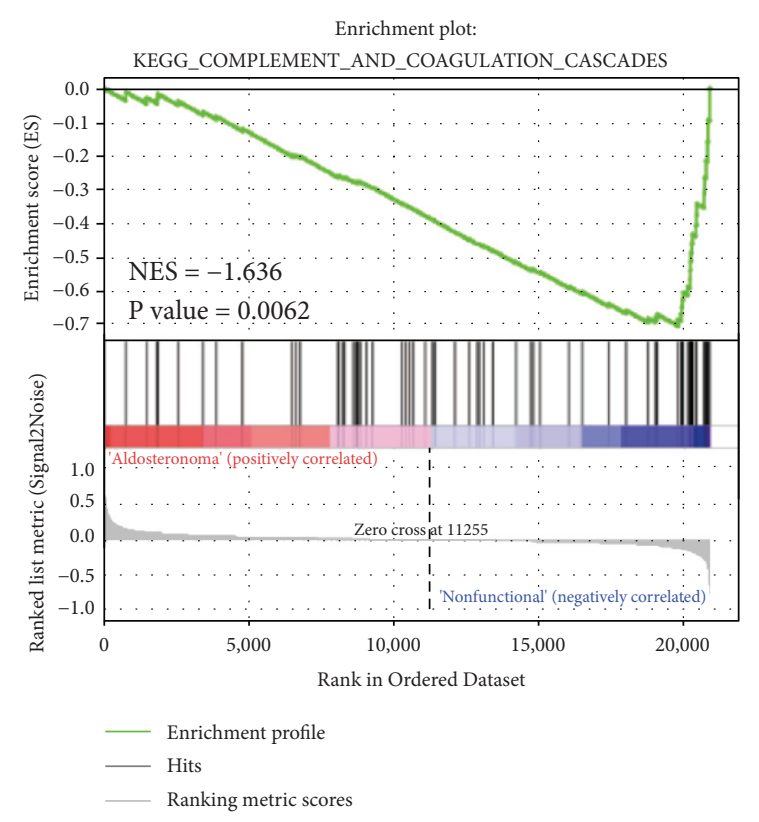

(b)

Figure 7: Continued. 


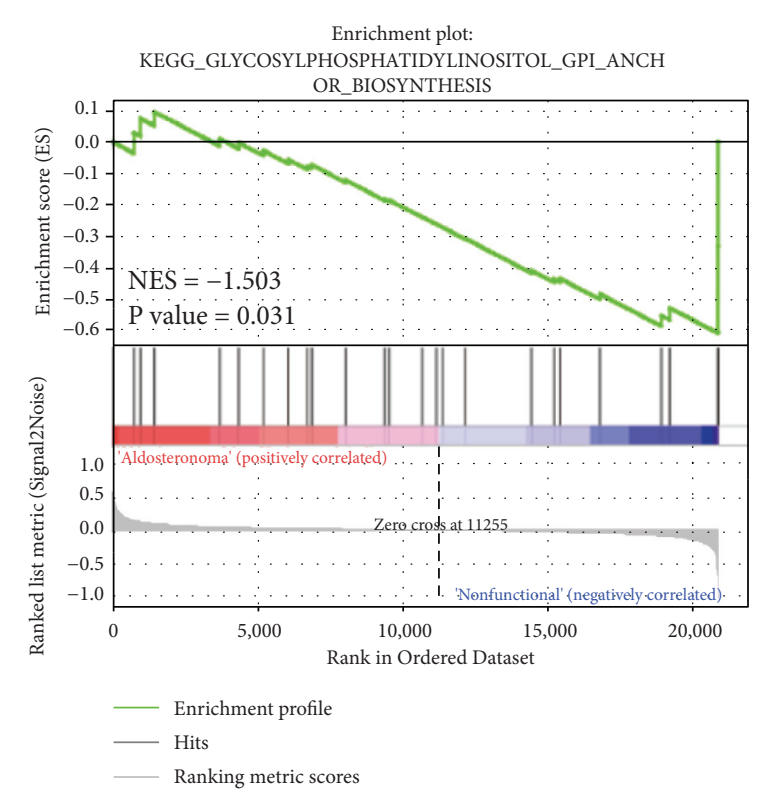

(c)

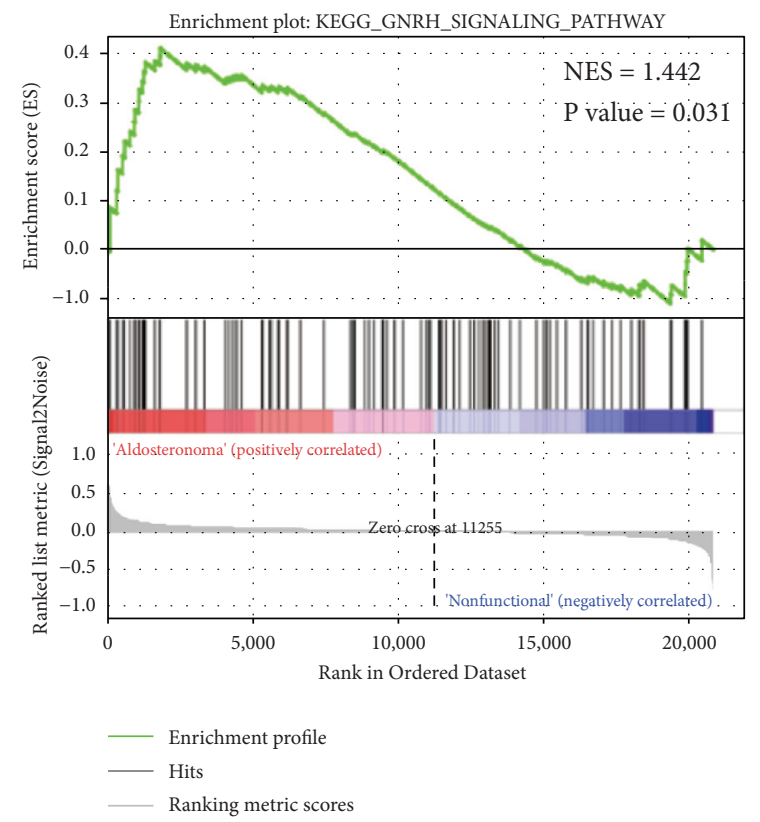

(e)

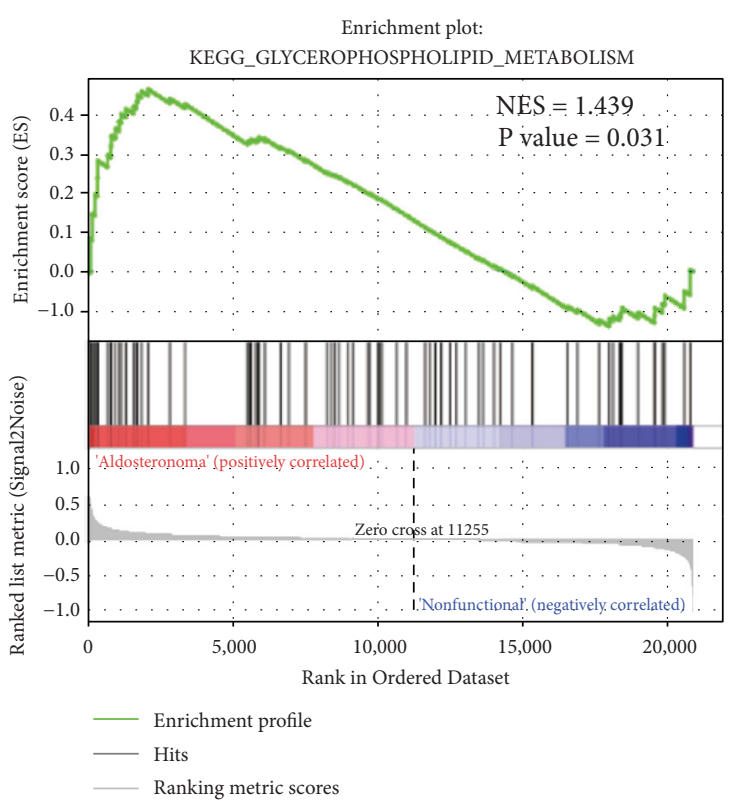

(d)

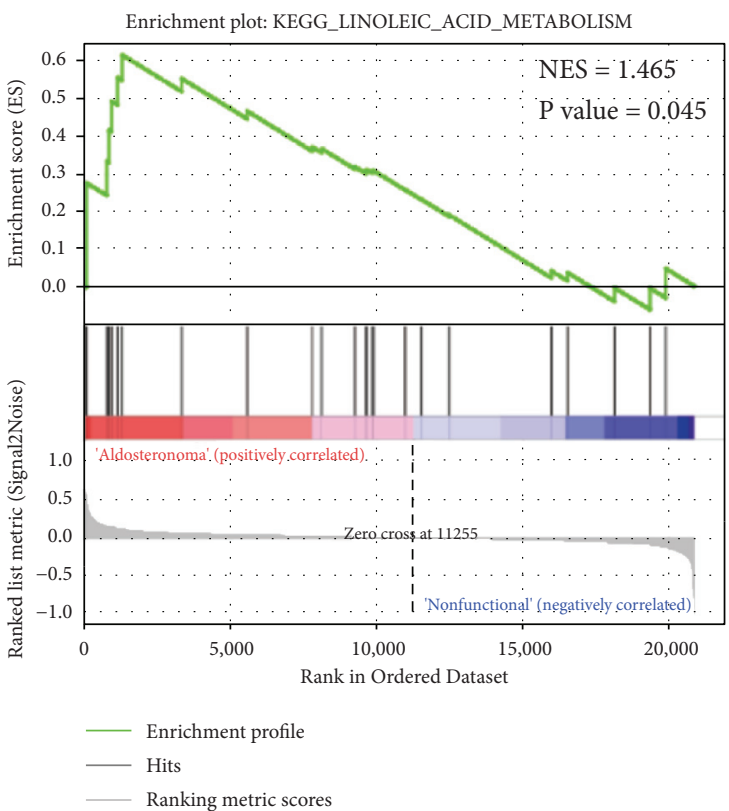

(f)

FIGURe 7: GSEA analysis for KEGG pathway enrichment using combined datasets. ABC, ATP-binding cassette; GPI, glycosylphosphatidylinositol; and GNRH, gonadotropin-releasing hormone.

false negative results during sample grouping process, it increased the specificity of the validation results, as only genes with a significant expression difference between APA and NFAA can be defined as DEG using the above screening criteria (Section 2).

Six genes-WISP2, S100A10, SSTR5-AS1, SLC29A1, $A P O C 1$, and SLITRK4-were identified having the same expression pattern in the combined and validation datasets. These genes play various roles in biological processes in which WISP2, S100A10, and APOC1 indirectly or directly participate in lipid metabolism. WISP2 is a novel adipokine, and it has been reported that WISP2 knockdown enhanced adipogenesis [25]. S100A10 is a cell membrane repair protein holding a complex interaction relationship with the phospholipid membrane [26]. SSTR5-AS1, an antisense lncRNA of SSTR5L, acts as a tumor suppressor, as well as a potential biomarker for antitumor therapy [27]. The human equilibrative nucleoside transporter 1 ( $h E N T 1)$, also called SLC29A1, a member of the SLC29 family, plays crucial roles in adenosine signaling, nucleoside-derived anticancer, and 

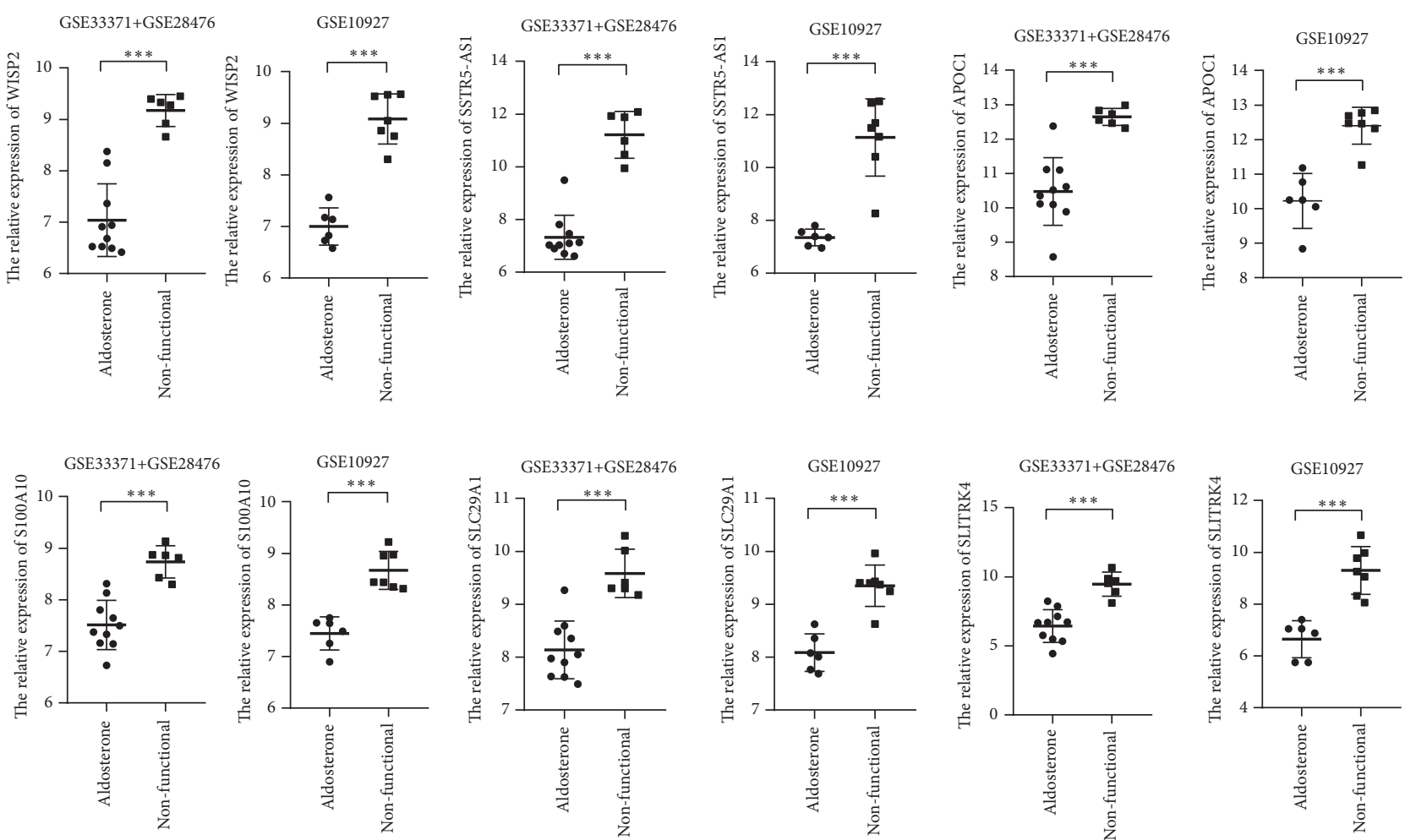

FIGURE 8: Validation of the expression pattern of real hub genes between the combined datasets (GSE333714 and GSE28476) and the validation dataset (GSE10927). Six genes, including one lncRNA gene, were found to have the same expression pattern between the combined and validation datasets. In addition, all of these genes were poorly expressed in the APA samples compared to the NFAA samples. The three asterisks represent $P$ values $<0.001$ between the two groups.

TABLE 1: The potential small-molecule drugs.

\begin{tabular}{|c|c|c|c|c|c|c|}
\hline Cmap name & Mean & $n$ & Enrichment score & $P$ value & Specificity & Percent nonnull \\
\hline Clorsulon & -0.588 & 4 & -0.843 & 0.00105 & 0.0071 & 100 \\
\hline Trimethoprim & -0.546 & 5 & -0.77 & 0.00118 & 0 & 100 \\
\hline Cycloheximide & -0.494 & 4 & -0.769 & 0.00571 & 0.0741 & 100 \\
\hline Meclocycline & -0.443 & 4 & -0.751 & 0.00772 & 0 & 75 \\
\hline Terfenadine & -0.575 & 3 & -0.839 & 0.00843 & 0.0642 & 100 \\
\hline
\end{tabular}

antiviral drug transport in humans [28]. Apolipoprotein C1 (APOC1), the smallest one in all of the apolipoproteins, participates in lipid transport and metabolism. It associates with triglyceride-rich lipoproteins and high-density lipoproteins and exchanges esterified cholesterol between lipoprotein classes, thereby controlling the plasma levels of lipids. Gautier's research suggests that human APOC1 transgenesis reduces atherogenesis in hypercholesterolemic rabbits [29]. APOC1 was also a key gene enriched, using the clusterProfiler package and Cytoscape (Tables 2 and 3), further confirming that phospholipid transportation and phospholipid metabolism may play an important role in the pathogenesis of APA.

Among all the candidate drugs speculated using the CMap database according to the gene expression file of DEGs, cycloheximide was the one that had been reported on suppressing adrenocortical activity [30]. Researchers had found that cycloheximide carried an inhibiting effect on the biosynthesis of total phospholipids [31]. Kempen's study suggested that cycloheximide not only suppresses very-lowdensity lipoprotein (VLDL) secretion but also inhibits the release of other apolipoproteins [32]. In an earlier study, phospholipid production was found to be severely inhibited in cycloheximide-treated cells [33]. A variety of evidence suggests that cycloheximide plays a role as an inhibitor for phospholipid biosynthesis. According to our study above, this countereffect of cycloheximide to phospholipid biosynthesis may be the pathophysiology basis of its inhibitory effect to APA. Whether the other candidate drugs also hold the properties like cycloheximide still needs more study to confirm. Furthermore, trimethoprim shows an ability to bind to the phospholipid matrix of the bilayer membrane [34], which indicates that trimethoprim may also be a potential effective drug for APA. 
<smiles>Nc1cc(C(Cl)=C(Cl)Cl)c(S(N)(=O)=O)cc1S(N)(=O)=O</smiles>

Clorsulon (C8H8Cl3N3O4S2)<smiles>COc1cc(Cc2cnc(NN)nc2NN)cc(OC)c1OC</smiles>

Trimethoprim (C14H18N4O3)<smiles>C[C@H]1C[C@H](C)C(=O)[C@H]([C@H](O)CC2CC(=O)NC(=O)C2)C1</smiles>

Cycloheximide (C15H23NO4)<smiles>C=C1c2c(Cl)ccc(O)c2C(O)=C2C(=O)[C@]3(O)C(O)=C(C(N)=O)C(=O)[C@@H](N(C)C)[C@H]3[C@H](O)[C@@H]12</smiles>

Meclocycline (C22H21ClN2O8)

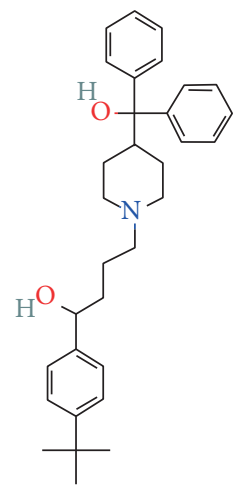

Terfenadine (C32H41NO2)

FIGURE 9: 2D structure of putative candidate small-molecule drugs against APA.

TABLE 2: GO enrichment analysis of real hub genes using the clusterProfiler package

\begin{tabular}{lccc}
\hline GO ID & GO terms & $q$ value & Gene symbol \\
\hline 0015914 & Phospholipid transport & 0.0274 & APOC1/ATP10A/ATP9A \\
0015748 & Organophosphate ester transport & 0.034 & APOC1/ATP10A/ATP9A \\
0045332 & Phospholipid translocation & 0.045 & ATP10A/ATP9A \\
0034204 & Lipid translocation & 0.045 & ATP10A/ATP9A \\
\hline
\end{tabular}

TABle 3: GO enrichment analysis of real hub genes using the Cytoscape software.

\begin{tabular}{lccc}
\hline GO ID & GO terms & q value & Gene symbol \\
\hline 15914 & Phospholipid transport & 0.004 & APOC1/ATP10A/ATP9A \\
6869 & Lipid transport & 0.042 & APOC1/ATP10A/ATP9A \\
10876 & Lipid localization & 0.042 & APOC1/ATP10A/ATP9A \\
42214 & Terpene metabolic process & 0.042 & $B C O 2$ \\
10899 & 0.042 & $A P O C 1$ \\
10900 & Regulation of the phosphatidylcholine catabolic process & 0.042 & APOC1 \\
\hline
\end{tabular}

\section{Conclusions}

Using differential gene expression analysis and WGCNA, we identified specific real hub genes for APA. Three of these genes encoded lncRNAs, and one of them encoded a transcription factor. We performed functional enrichment analysis using these real hub genes. Most of the enrichment items were related to phospholipid metabolism. Additionally, we found out some promising candidate drugs for the treatment of APA using the CMap database, one of which had been reported on suppressing adrenocortical activity and was also a potent inhibitor for phospholipid 
biosynthesis. These results suggest that phospholipid metabolism is an important mechanism that may control aldosterone production and (or) secretion from APA. Therefore, drugs with the ability to regulate the phospholipid metabolic process on adrenal tissue may be promising medications for APA. However, the number of samples used in this study was small, a limitation which might bias our conclusions. Hence, more studies with larger sample sizes are required in future.

\section{Data Availability}

The datasets generated and/or analyzed during the current study are available from the Gene Expression Omnibus data repository (https://www.ncbi.nlm.nih.gov/geo/) with the accession numbers GSE33371, GSE28476, and GSE10927.

\section{Conflicts of Interest}

The authors declare no conflicts of interest.

\section{Authors' Contributions}

YYD contributed to manuscript writing. JL offered great help for the revisions of manuscript and provided the guidance for English language editing and data analysis and methodology design. HW participated in partial data analysis. JLL contributed to the design and conception of this study. All authors have read and approved the final manuscript.

\section{Acknowledgments}

The authors thank Mr. Qisheng Su from the Department of Clinical Laboratory, The First Affiliated Hospital of Guangxi Medical University, for his guidance during this study. This study was supported by the National Natural Science Foundation of China (Nos. 81660075 and Nos. 81960087) and the Guangxi Medical and Health Appropriate Technology Development and Promotion Application Project (Nos. S2018 078).

\section{References}

[1] J. B. Cohen, D. L. Cohen, D. S. Herman, J. T. Leppert, J. B. Byrd, and V. Bhalla, "Testing for primary aldosteronism and mineralocorticoid receptor antagonist use among U.S. Veterans," Annals of Internal Medicine, vol. 174, no. 3, pp. 289-297, 2021.

[2] S. Monticone, F. D’Ascenzo, C. Moretti et al., "Cardiovascular events and target organ damage in primary aldosteronism compared with essential hypertension: a systematic review and meta-analysis," The Lancet Diabetes \& Endocrinology, vol. 6, no. 1, pp. 41-50, 2018.

[3] J. Funder, "Primary aldosteronism," Trends in Cardiovascular Medicine, 2021, inpress.

[4] E. Lalli, J. Barhanin, M.-C. Zennaro, and R. Warth, "Local control of aldosterone production and primary aldosteronism," Trends in Endocrinology and Metabolism, vol. 27, no. 3, pp. 123-131, 2016.
[5] T. Ichijo, H. Ueshiba, H. Nawata, and T. Yanase, "A nationwide survey of adrenal incidentalomas in Japan: the first report of clinical and epidemiological features," Endocrine Journal, vol. 67, no. 2, pp. 141-152, 2020.

[6] M. Choi, U. I. Scholl, and P. Yue, "K+ channel mutations in adrenal aldosterone-producing adenomas and hereditary hypertension," Science (New York, NY), vol. 331, no. 6018, pp. 768-772, 2011.

[7] U. I. Scholl, G. Goh, G. Stölting et al., "Somatic and germline CACNA1D calcium channel mutations in aldosterone-producing adenomas and primary aldosteronism," Nature Genetics, vol. 45, no. 9, pp. 1050-1054, 2013.

[8] G. P. Rossi, "A comprehensive review of the clinical aspects of primary aldosteronism," Nature Reviews Endocrinology, vol. 7, no. 8, pp. 485-495, 2011.

[9] L. Amar, A. Lorthioir, M. Azizi, and P.-F. Plouin, "Progress IN primary aldosteronism: mineralocorticoid antagonist treatment for aldosterone-producing adenoma," European Journal of Endocrinology, vol. 172, no. 3, pp. R125-R129, 2015.

[10] Y. Tezuka and A. F. Turcu, "Mineralocorticoid receptor antagonists decrease the rates of positive screening for primary aldosteronism," Endocrine Practice, vol. 26, no. 12, pp. 1416-1424, 2020.

[11] J. Durand, A. Lampron, T. L. Mazzuco, A. Chapman, and I. Bourdeau, "Characterization of differential gene expression in adrenocortical tumors harboring $\beta$-catenin (CTNNB1) mutations," Journal of Clinical Endocrinology \& Metabolism, vol. 96, no. 7, pp. E1206-E1211, 2011.

[12] J. H. Heaton, M. A. Wood, A. C. Kim et al., "Progression to adrenocortical tumorigenesis in mice and humans through insulin-like growth factor 2 and $\beta$-catenin," American Journal Of Pathology, vol. 181, no. 3, pp. 1017-1033, 2012.

[13] T. J. Giordano, R. Kuick, T. Else et al., "Molecular classification and prognostication of adrenocortical tumors by transcriptome profiling," Clinical Cancer Research, vol. 15, no. 2, pp. 668-676, 2009.

[14] R. A. Irizarry, B. Hobbs, F. Collin et al., "Exploration, normalization, and summaries of high density oligonucleotide array probe level data," Biostatistics, vol. 4, no. 2, pp. 249-264, 2003.

[15] J. T. Leek, W. E. Johnson, H. S. Parker, A. E. Jaffe, and J. D. Storey, "The sva package for removing batch effects and other unwanted variation in high-throughput experiments," Bioinformatics, vol. 28, no. 6, pp. 882-883, 2012.

[16] P. Langfelder and S. Horvath, "WGCNA: an R package for weighted correlation network analysis," BMC Bioinformatics, vol. 9, p. 559, 2008.

[17] P. Shannon, A. Markiel, and O. Ozier, "Cytoscape: a software environment for integrated models of biomolecular interaction networks," Genome Research, vol. 13, no. 11, pp. 2498-2504, 2003.

[18] G. Yu, L.-G. Wang, Y. Han, and Q.-Y. He, "clusterProfiler: an $\mathrm{R}$ Package for comparing biological themes among gene clusters," OMICS: A Journal of Integrative Biology, vol. 16, no. 5, pp. 284-287, 2012.

[19] A. Subramanian, P. Tamayo, V. K. Mootha et al., "Gene set enrichment analysis: a knowledge-based approach for interpreting genome-wide expression profiles," Proceedings of the National Academy of Sciences, vol. 102, no. 43, pp. 15545-15550, 2005.

[20] J. Lamb, E. D. Crawford, and D. Peck, “The Connectivity Map: using gene-expression signatures to connect small molecules, genes, and disease," Science, vol. 313, no. 5795, pp. 1929-1935, 2006. 
[21] X. Su, J. Zhang, X. Luo et al., "LncRNA LINC01116 promotes cancer cell proliferation, migration and invasion in gastric cancer by positively interacting with lncRNA CASC11," OncoTargets and Therapy, vol. 12, pp. 8117-8123, 2019.

[22] T. Wang, L. Cao, X. Dong et al., "LINC01116 promotes tumor proliferation and neutrophil recruitment via DDX5-mediated regulation of IL-1 $\beta$ in glioma cell," Cell Death \& Disease, vol. 11 , no. 5 , p. $302,2020$.

[23] J. Wu, Z. Chen, L. Zhang et al., "Knockdown of LINC01116 inhibits cell migration and invasion in head and neck squamous cell carcinoma through epithelial-mesenchymal transition pathway," Journal of Cellular Biochemistry, vol. 121, no. 1, pp. 867-875, 2020.

[24] N. Endlich, F. Kliewe, F. Kindt et al., "The transcription factor Dach1 is essential for podocyte function," Journal of Cellular and Molecular Medicine, vol. 22, no. 5, pp. 2656-2669, 2018.

[25] L. Ren, Q. Li, X. Hu et al., "A novel mechanism of bta-miR-210 in bovine early intramuscular adipogenesis," Genes, vol. 11, no. 6,2020

[26] X. Yan, K. Kumar, and R. Miclette Lamarche, "Interactions between the cell membrane repair protein S100A10 and phospholipid monolayers and bilayers," Langmuir: The ACS Journal of Surfaces and Colloids, vol. 37, 2021.

[27] B. Wang, L. Zhao, W. Chi, H. Cao, W. Cui, and W. Meng, "Aberrant methylation-mediated downregulation of lncRNA SSTR5-AS1 promotes progression and metastasis of laryngeal squamous cell carcinoma," Epigenetics \& Chromatin, vol. 12, no. 1, p. 35, 2019.

[28] N. J. Wright and S.-Y. Lee, "Structures of human ENT1 in complex with adenosine reuptake inhibitors," Nature Structural \& Molecular Biology, vol. 26, no. 7, pp. 599-606, 2019.

[29] T. Gautier, V. Deckert, V. Aires et al., "Human apolipoprotein C1 transgenesis reduces atherogenesis in hypercholesterolemic rabbits," Atherosclerosis, vol. 320, pp. 10-18, 2021.

[30] D. Szabó, I. E. Tóth, and K. S. Szalay, "Viscosity of rat adrenocortical lipids in different functional states: morphological characteristics," The Journal of Steroid Biochemistry and Molecular Biology, vol. 58, no. 3, pp. 329-335, 1996.

[31] M. Vasileva-Dimova, "The effect of adrenocorticotropic hormone (ACTH) and cycloheximide (CHM) on lipid biosynthesis in the rat lung," Eksperimentalna meditsina $i$ morfologiia, vol. 29, no. 2, pp. 63-67, 1990.

[32] H. J. Kempen, "Lipoprotein secretion by isolated rat hepatocytes: characterization of the lipid-carrying particles and modulation of their release," Journal of Lipid Research, vol. 21, no. 6, pp. 671-680, 1980.

[33] D. G. Bishop and R. M. Smillie, "The effect of chloramphenicol and cycloheximide on lipid synthesis during chloroplast development in Euglena gracilis," Archives of Biochemistry and Biophysics, vol. 139, no. 1, pp. 179-189, 1970.

[34] G. R. Painter, R. Grunwald, and B. Roth, "Interaction of the antifolate antibiotic trimethoprim with phosphatidylcholine membranes: a $13 \mathrm{C}$ and $31 \mathrm{P}$ nuclear magnetic resonance study," Molecular Pharmacology, vol. 33, no. 5, pp. 551-558, 1988. 